\title{
The effect of educational intervention based on an Ecological-social model on consuming fruit and vegetables in women in Ilam
}

\author{
Leili Shafiei $^{1}$, Parvaneh Taymoori ${ }^{2,3}$, Afshin Maleki $^{1}$, Kourosh Sayehmiri $^{4}$
}

${ }^{1}$ Environmental Health Research Center, Kurdistan University of Medical Sciences, Sanandaj, Iran

${ }^{2}$ Social Determinants of Health Research Center, Kurdistan University of Medical Sciences, Sanandaj, Iran

${ }^{3}$ Department of Public Health, Faculty of Health, Kurdistan University of Medical Sciences, Sanandaj, Iran

${ }^{4}$ Psychosocial Injuries Research Center, Biostatistics department, Ilam University of Medical Sciences, Ilam, Iran

\section{Type of article: Original}

\begin{abstract}
Background: The effect of instructional models on the changing behavior of consuming fruit and vegetables in the prevention of diseases caused by consumption of foods including rice, contaminated with toxic metals, has not been investigated in Iran yet.

Objective: To compare an Ecological-social model (ECO) group and Control group in increasing fruit and vegetable consumption.

Methods: This research involved implementation of a six-month randomized controlled educational interventionist program into a group of Ecological-social framework along with a control group, totally summing up to 160 women between 18 and 50 years of age in Ilam, Iran in 2014. The questionnaire included knowledge, social support construct (immediate family, relatives, friends, colleagues and neighbors.) and fruit and vegetable intake in both groups before and after the intervention was examined. Data were analyzed by SPSS version 16 using the signed ranks test, and repeated measures analysis of variance analysis, and confidence interval of $95 \%$ were employed.

Results: The results of the signed ranks test showed a significant increase in social support in the intervention group $(\mathrm{p}<0.001)$. This test showed a significant increase in fruit and vegetable consumption and a significant increase in the number of women using healthy rice $(\mathrm{p}<0.001)$, and a significant reduction in the number of women consuming unhealthy rice in the intervention group $(\mathrm{p}<0.001)$, that determines dietary intake improvement after the intervention compared with before the intervention $(\mathrm{p}<0.01)$. However, this improvement was not observed in the control group.

Conclusions: The results showed that ECO plays an effective role in improving fruit and vegetable consumption in women. Therefore, this model was implemented as a health protocol through health-care centers to conduct the prevention of complications resulting from the use of food contaminated with toxic metals.

Keywords: Educational intervention, Heavy Metal Toxicity, Ecological-social model, Fruit, Vegetables
\end{abstract}

\section{Introduction}

Rice is the second most widely consumed food in Iran (1). On the other hand, due to the presence of toxic metals in some rice consumed by households in Iran $(2,3)$, side effects caused by their continuing consumption such as cancer and their damaging impacts on lung, liver, nervous system and lungs are not unexpected (4). Fruit and vegetable consumption, due to high amounts of antioxidants, can prevent chronic diseases, especially cancer (5). Studies show the inverse relationship between consumption of fruits and vegetables and cancer $(6,7)$, in addition to the potential role of antioxidants as treatment in toxic metal exposure proved (8). The World Health Organization (WHO) has recommended eating at least 3-5 units of fruit and vegetables per day (9). According to a study conducted in 21 countries, the fruit and vegetable consumption pattern has only been met in three countries (10) and in many countries such as Portugal, America, Belgium and Iran it is less than the recommended amount (11). Therefore, increasing the consumption of fruit and vegetables can reduce the complications of rice contaminated with toxic

\section{Corresponding author:}

Parvaneh Taymoori. Tel: +98-9183737303, Fax:+98-871-6131468, Email: Parvaneh.tay@gmail

Received: April 05, 2017, Accepted: July 27, 2017, Published: December 2017

iThenticate screening: June 17, 2017, English editing: September 22, 2017, Quality control: October 26, 2017

(C) 2017 The Authors. This is an open access article under the terms of the Creative Commons Attribution-NonCommercialNoDerivs License, which permits use and distribution in any medium, provided the original work is properly cited, the use is non-commercial and no modifications or adaptations are made. 
metals including lead, cadmium, and arsenic in the body. A person's food habits are directly or indirectly affected by the person's social environment (12). One of the successful models used in adherence to a healthy diet and intake of fruits and vegetables is the Ecological-Social model (ECO) (13). In the ECO the relationship between individual, social and environmental factors and fruit and vegetable consumption has been confirmed (14). Lack of social support is the main barrier to change in food habits, weight loss, healthy eating and healthy lifestyle $(15,16)$. Several studies considered the worksite as an appropriate behavioral model to increase consumption of fruits and vegetables $(13,17)$. Also, there is empirical evidence of the predictive role of social support in increased consumption of fruits and vegetables (13), behavior due to reduced consumption of unhealthy food (18) and weight loss (19). Several studies have been conducted on the role of the ECO framework in changing nutrition behavior of people $(13,17)$. But so far, no studies have been conducted on whether the ECO model in encouraging people to consume fruit and vegetables to prevent and mitigate the effects of toxic metals in the body is effective or not. In this study, it was assumed that social networking and attending the role of family members, friends, and colleagues would have a significant impact on household consumption of fruit and vegetables in the prevention of diseases caused by the consumption of rice contaminated with toxic metals. Testing this hypothesis will lead to identify strategies of preparation to improve diet. The gap in previous studies was filled by testing the fact that, the intervention model ECO compared with the control group underlies the improvement in consumption of fruit and vegetables by Iranian women. In this intervention model, people were involved through researchers and social network including family members, friends, and colleagues. Additionally, considering the role of women in families and their impact in the application of educational- health patterns (20), and availability of local rice for women in Ilam, this quasi-experimental study was conducted in the offices of Ilam. The study assumptions included: 1) the difference between before and after the intervention in the ECO group in knowledge, social support construct is significant; and 2) there is a significant difference between before and after the intervention in ECO and control groups, and this difference in increasing fruit and vegetable consumption and decreased rice contaminated with toxic metals in group ECO is higher than the control group.

\section{Material and Methods}

\subsection{Research design}

This study is of an educational intervention program based on ECO in both the intervention and control groups in Ilam, Iran in 2014. Inclusion criteria in this study were women aged 18 to 50 years with the responsibility of cooking, and who voluntarily agreed to participate in the study. Exclusion criteria was dissatisfaction with participating in training sessions. To obtain the probability of women's awareness of fruit and vegetable consumption as means of decreasing the effects of toxic metals on the body using pre-test questionnaire, 30 subjects with the characteristics of the target population were studied and the knowledge of $30 \%$ was obtained. For determining sample size, $\alpha=0.05$ and $\beta=20 \%$ were considered and estimating minimum increased awareness expected of at least $20 \%$ after the intervention, the number of samples of 80 for each of the groups (intervention group and control group), a total of 160 was obtained. Then among 12 offices of Ilam, 6 were randomly considered.

\subsection{Data collection}

Data collection was conducted in two stages, before (December 2014) and after the intervention (June 2015). The outcome measured was the performance of fruit and vegetable consumption per day and consumption of healthy (local rice) and unhealthy rice (foreign rice).

\subsection{Instruments}

The survey instrument used in this study was a questionnaire with 17 questions and 3 parts, including knowledge, social support constructs and performance of the consumption of fruits and vegetables. Knowledge of fruit and vegetable consumption as a strategy of decreasing the effects of toxic metals in the body was determined as a question of "Do you have knowledge or not?", as the frequency. Social support includes 9 questions on social networks support of individuals separated by the mother, father, sister, brother, wife, children, friends and colleagues, neighbors and family members about fruits and vegetables consumption to reduce the effects of toxic metals in the body. The responses were evaluated as three items (often $=2$ to never $=0$ ). The score of this construct was considered by calculating the average as social support score. Cronbach's alpha coefficient obtained in the social support sector was 0.88 . The score range of 0 to 18 was the total social support of social network members. The reliability of the performance obtained by test was $\mathrm{K}=0.85$. Water and Food Research Center, Kurdistan University of Medical Sciences approved the protocol of intervention plan of this design. 


\subsection{Planning of Intervention}

For the data collection, the interviewer visited the women at their worksite, and for the control group, in their homes. Before completing the questionnaires, a brief description of the study and implementation of training sessions for participants were described. After completing the questionnaire, based on the ECO model as the initial assessment and after complete satisfaction, 45 to 60-minute training sessions in offices as lecture, group discussion and individual counseling were held, for the intervention group. No training sessions were held for women in the control group. In the first session, descriptions in order to increase public awareness of environmental pollutants, including toxic metals, rice contaminated with toxic metals and the benefits of eating fruit and vegetables in the diet as the strategies of decreasing the effects of toxic metals in the body were provided. In this session, considering the contamination of foreign rice, local rice of Ilam was introduced as safe rice free of toxic metals. In the second session, small groups in offices were established to discuss the consumption of fruit and vegetables and healthy rice consumption. In the third session, the social network of employees was expended by adding mother, sister, friends, colleagues, and family members in virtual spaces such as Viber, WhatsApp, and Telegram. In these virtual groups, content of training sessions was given to the social network of people, and discussion took place in the same group. In the fourth session, an individual session was held with each female employee with an emphasis on increasing fruit and vegetable consumption and consumption of healthy rice and overcoming the barriers of consumption of rice contaminated with toxic metals.

\subsection{Data Entry and Statistical Analysis}

After evaluating the normality of data by Kolmogorov-Smirnov test in SPSS version 16 (SPSS Inc., Chicago, Illinois, USA), the descriptive analysis of the variables, and descriptive statistics of the frequency, mean and confidence intervals of $95 \%$ were used. In performance, the consumption of fruit and vegetables per day and the frequency of female consumers of healthy and unhealthy rice was evaluated. Nonparametric signed ranks test was used to assess changes in social support construct and performance of fruit and vegetable consumption in both ECO and control groups from before to after the intervention. The repeated measures analysis of variance to compare fruit and vegetable consumption in both ECO and control groups before and after the intervention was used. Significance level of $\mathrm{P}<0.05$ was considered.

\section{Results}

The average age of women participating in the ECO group was $33.76 \pm 5.24$ and in the control group it was $36.4 \pm 9$ years. In the ECO group, $64 \%$ of women and $73 \%$ of women in the control group were married. A significant increase in knowledge and social support after the intervention was observed compared with before the intervention in the group ECO $(\mathrm{p}<0.01)$. Social support scores of mother, father, sister, brother, wife, children, friends and colleagues, neighbors and family members in the intervention group after the educational program were respectively, $1.26,1,1.15,0.93,1.12,0.27,2,0.83$ and 1.2 , while the control group did not receive any support in order to increase fruits and vegetables. In addition, a significant increase in the consumption of fruits and vegetables was observed after the intervention compared with before the intervention. Increasing the proportion of women using healthy rice and reduced frequency of women consuming unhealthy rice in ECO group were significant $(\mathrm{p}<0.01)$ (Table 1).

Table 1. The performance of consumption of fruit and vegetables, healthy and unhealthy rice in ECO and control groups before and after intervention

\begin{tabular}{|c|c|c|c|c|c|c|c|}
\hline \multirow{2}{*}{\multicolumn{2}{|c|}{ Variables }} & \multicolumn{3}{|c|}{$\mathrm{ECO} ; \mathrm{n}(\%) / \mathrm{Mean} \pm \mathrm{SD}$} & \multicolumn{3}{|l|}{ Control } \\
\hline & & $\begin{array}{l}\text { Before } \\
\text { intervention }\end{array}$ & $\begin{array}{l}\text { After } \\
\text { intervention }\end{array}$ & $\begin{array}{l}\mathrm{p}- \\
\text { value }\end{array}$ & $\begin{array}{l}\text { Before } \\
\text { intervention }\end{array}$ & $\begin{array}{l}\text { After } \\
\text { intervention }\end{array}$ & $\begin{array}{l}\mathrm{p}- \\
\text { value }\end{array}$ \\
\hline \multirow[t]{2}{*}{ Knowledge } & Yes & $46(57.5)$ & $80(100)$ & \multirow[t]{2}{*}{0.000} & $12(15)$ & $4(5)$ & \multirow[t]{2}{*}{0.033} \\
\hline & No & $34(42.5)$ & 0 & & $68(85)$ & $76(95)$ & \\
\hline \multicolumn{2}{|c|}{ Social support } & 0 & $9.8 \pm 3.4$ & 0.000 & 0 & 0 & 1.00 \\
\hline \multicolumn{2}{|c|}{$\begin{array}{l}\text { Frequency of women } \\
\text { consuming healthy rice }\end{array}$} & $2(2.5)$ & $26(32.5)$ & \multirow[t]{2}{*}{0.000} & $9(11.2)$ & 0 & \multirow[t]{2}{*}{0.003} \\
\hline \multicolumn{2}{|c|}{$\begin{array}{l}\text { Frequency of women } \\
\text { consuming unhealthy rice }\end{array}$} & $70(87.5)$ & $54(67.5)$ & & $71(88.8)$ & $80(100)$ & \\
\hline \multicolumn{2}{|c|}{$\begin{array}{l}\text { Number consumption of fruit } \\
\text { per day }\end{array}$} & $1.54 \pm 0.84$ & $2.03 \pm 0.94$ & 0.000 & $0.81 \pm 0.64$ & $0.69 \pm 0.98$ & 0.363 \\
\hline \multicolumn{2}{|c|}{$\begin{array}{l}\text { Number consumption of } \\
\text { vegetables per day }\end{array}$} & $0.5 \pm 0.92$ & $1.22 \pm 0.73$ & 0.000 & $0.68 \pm 0.52$ & $0.04 \pm 0.33$ & 0.000 \\
\hline \multicolumn{2}{|c|}{$\begin{array}{l}\text { Number consumption of fruit \& } \\
\text { vegetables per day }\end{array}$} & $2.03 \pm 1.24$ & $3.25 \pm 1.23$ & 0.000 & $1.48 \pm 0.99$ & $0.72 \pm 1.01$ & 0.000 \\
\hline
\end{tabular}


Statistical analysis repeated measures analysis of variance showed also that the two groups had significant differences in consumption of fruit and vegetables $(p<0.05)$. As after the intervention the consumption of fruit and vegetable in the ECO group was higher than the control group but in the control group consumption of fruit and vegetables decreased (Figure 1).

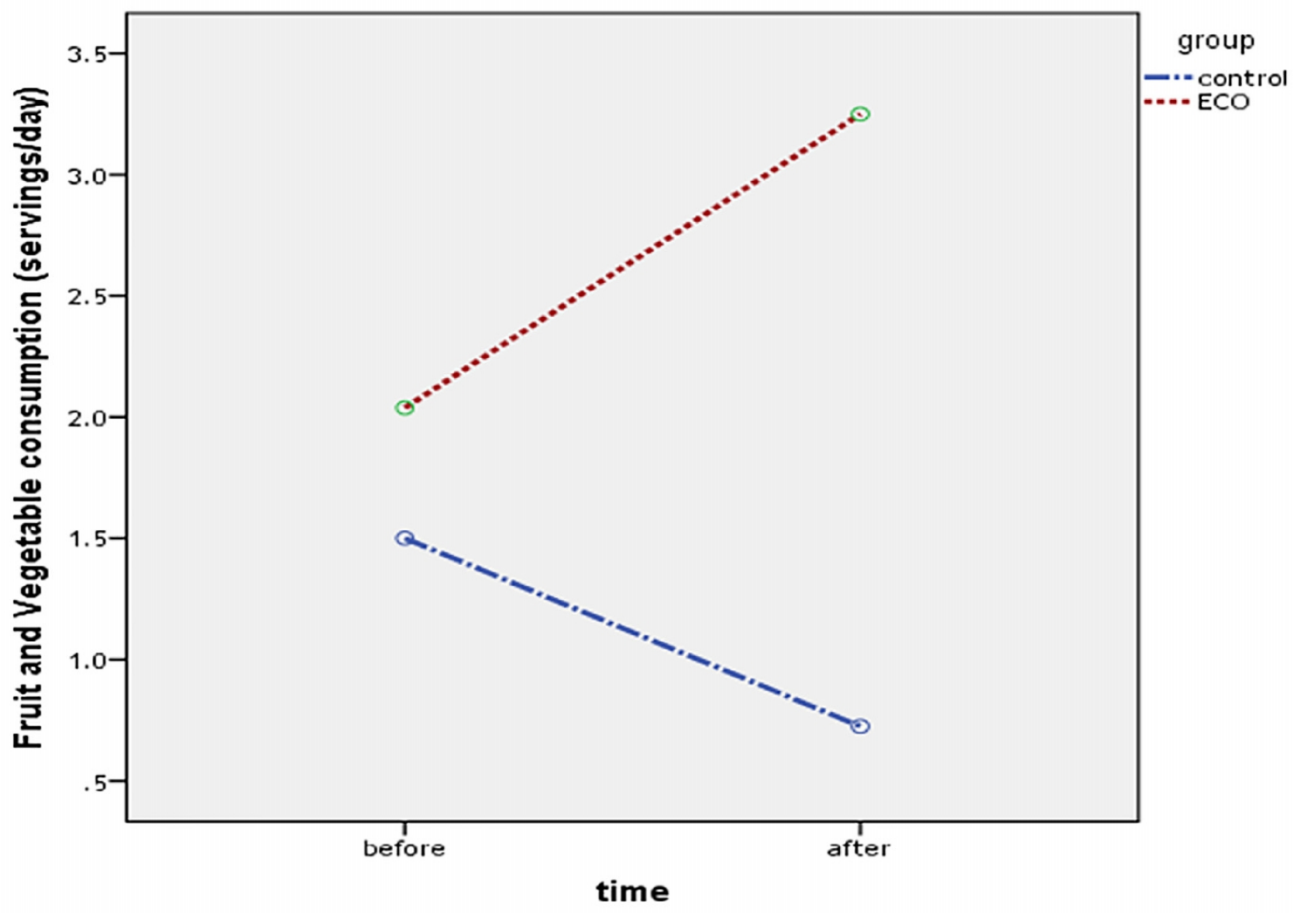

Figure 1. Changes in consumption of fruit $\&$ vegetables in terms of frequency of consumption per day in the two groups (ECO and control) before and after the intervention

\section{Discussion}

This is the first study of a randomized controlled educational intervention plan to compare the effect of an interventional model of ECO on fruit and vegetable intake to prevent people from contracting diseases from consumption of rice contaminated by toxic metals. In the present study, women's knowledge in the intervention group increased. This increase was due to training and educational sessions in the experimental group. There were positive changes in the construction of social support in the intervention group compared with the control group. These positive changes in the intervention group were due to the implementation of training programs, increasing social networks and gaining awareness of the impact of fruit and vegetable intake in reducing the effects of toxic metals in the body compared to the control group. Most support was for fruit and vegetable consumption by colleagues because of social networking at their worksite. Our work was similar to a 2013 study by Geaney et al., on employees' dietary intake in manufacturing workplaces in Ireland to increase the nutritional knowledge (21) and a 2005 study by Tamers et al. on 2,878 employees from 34 worksites through changes in eating to increase social support in the worksite in order to increase the consumption of fruit and vegetables (22). It was assumed that the ECO model-based intervention played a role in the increase of fruit and vegetable intake for women. The key point of the intervention program was the introduction of consumption of fruit and vegetables due to high levels of antioxidants as a key strategy in reducing the effects of toxic metals in the body. So, the evaluation of abundance changes in fruit and vegetable consumption was considered as the outcome of the interventional program. The featured result in this study was the increased consumption of fruit and vegetables (per day) in the ECO group compared to the control group. Therefore, the ECO group had a healthier diet than the control group. In other words, ECO women consumed more fruit and vegetables as compared to the control group as a result of more social support. Furthermore, these results in terms of the effect of the ECO in improving fruit and vegetable consumption in the intervention group were similar to previous findings $(13,23)$. Due to the lack of an educational program in the control group before and after intervention, they did not receive any social support for fruit and vegetable consumption. The strength in this study was the received educational materials by the control group after completing the training program in the intervention group. The results showed that, focus on the worksite is effective in 
increasing the consumption of healthy foods $(13,22)$. The educational intervention based on ECO, increased the amount of fruits and vegetables by $15 \%$, while no change was observed in the control group (13). On the other hand, in the ECO group, the number of women who consumed healthy rice in their diet compared to the control group, increased after the intervention. However, this number decreased in the control group. The number of women consuming unhealthy rice in the ECO group decreased after the intervention and this number increased in the control group. The results in reduced consumption of unhealthy foods (24) and increased consumption of healthy foods were similar to previous studies $(24,25)$. The factor that led to achievements in improving fruit and vegetable intake and decreased unhealthy rice consumption in the ECO group more than the control group was enhanced social support by mother, father, sister, brother, spouse, children, relatives and neighbors, especially colleagues, and in other words social network development for fruit and vegetable consumption. So, one of the ways to successfully improve the performance of nutritional programs in the future is to consider social support and expansion of social networks.

\section{Limitations}

Implementation of educational intervention with regard to the extension of social network and culture of Ilam in ordinary women was not operational; thus, it can lead to bias in the results. However, given that in both groups, factors before and after the intervention were measured, therefore, this issue could reduce bias. On the other hand, due to the limitations in implementation of the educational program in the houses of people to form a social network consisting of neighbors, due to the cultural setting of the region, employees employed in organizations were chosen due to convenience in holding educational sessions and forming social networks. The performance of employees, due to their higher academic levels when compared with the control group, had better progress and the effect of academic levels was not considered in evaluation of the performance of rice consumption. Despite the training provided, considering the low financial power of households, some people are not able to increase consumption of fruit and vegetables and healthy rice due to the high price.

\section{Conclusions}

Although the educational intervention program based on the ecological model has led to an increase in fruit and vegetable consumption, on the other hand, it has also improved dietary intake in increasing consumption of toxic metals free rice and reduced use of rice contaminated to toxic metals. The increased social support will have a positive impact in improving people's diets. This is the first evidence of an investigation of the effects of community-based intervention (ECO model) taking into account the effects of social support on increasing fruit and vegetable consumption in order to prevent diseases from consumption of food contaminated with toxic metals.

\section{Acknowledgments:}

This paper was a result of a thesis called "Investigation of the effect of interventions based on the Health Belief Model and Ecological-Social framework on consumption of rice contaminated with toxic metals" for PhD by research associated with Ms. Leili Shafiei, approved in Kurdistan University of Medical Sciences.

\section{Conflict of Interest:}

There is no conflict of interest to be declared.

\section{Authors' contributions:}

All authors contributed to this project and article equally. All authors read and approved the final manuscript.

\section{References:}

1) Khaniki GR, Zozali MA. Cadmium and lead contents in rice (oryza sativa) in the north of Iran. Int J Agric Biol. 2005; 6: 1026-9.

2) Zazouli MA, Shokrzadeh M, Izanloo H, Fathi S. Cadmium content in rice and its daily intake in Ghaemshahr region of Iran. Afr J Biotechnol. 2008; 7(20): 3686-9.

3) Falahi E, Hedaiati R, Ghiasvand AR. Survey of iron, zinc, calcium, copper, lead and cadmium in rice samples grown in Iran. Food Additives Contaminants: Part B: Surveillance. 2010; 3(2): 80-3. doi: $10.1080 / 19440041003671288$.

4) Järup L. Hazards of heavy metal contamination. Br Med Bull. 2003; 68(1): 167-82. PMID: 14757716.

5) Wilson T. Nutritional health strategies for disease prevention. Springer. 2001: 16-93.

6) Steinmetz KA, Potter JD. Vegetables, fruit, and cancer prevention: A review. Am Diet Assoc. 1996; 96(10): 1027-39. doi: 10.1016/S0002-8223(96)00273-8. PMID: 8841165. 
7) Johnston CS, Taylor CA, Hampl JS. More Americans are eating 5 a day but intakes of dark green and cruciferous vegetables remain low. J Nutr. 2000; 130(12): 3063-7. PMID: 11110870.

8) Patrick L. Toxic metals and antioxidants: part II the role of antioxidants in arsenic and cadmium toxicity Toxic Metals Part II. Altern Med Rev. 2003; 8(2): 106-28. PMID: 12777158.

9) World Health Organization. Diet, nutrition and the prevention of chronic diseases. Report of a Joint WHO/FAO Expert consultation. Geneva: World Health Organization; 2003.

10) International Agency for Research on Cancer. IARC Handbook of Cancer Prevention. France: Lyon, IARC Press; 2003.

11) Piraghaj M, Amini M, Hooshyar Rad A, Abdollahi M, Zoghi T, Eslami amirabadi M. Qualitative and Quantitative Dietary Assessment of primary school students in Tehran. Iranian J Nutr Sci \& Food Technol. 2008; 3(1): 31- 44.

12) Feunekes GI, Stafleu A, de Graaf $C$, van Staveren WA. Family resemblance in fat intake in the Netherlands. Eur J clin Nutr. 1997; 51: 793-9. doi: 10.1038/sj.ejcn.1600494. PMID: 9426352.

13) Bandoni DH, Sarno F, Jaime PC. Impact of an intervention on the availability and consumption of fruits and vegetables in the workplace. Public Health Nutr. 2010; 14(6): 975-81. doi: 10.1017/S1368980010003460. PMID: 21205408.

14) Graham DJ, Pelletier JE, Neumark-Sztainer D, Lust K, Laska MN. Perceived Social-Ecological Factors Associated with Fruit and Vegetable Purchasing, Preparation, and Consumption among Young Adults. J Acad Nutr Diet. 2013; 113(10): 1366-74. doi: 10.1016/j.jand.2013.06.348. PMID: 23958116, PMCID: PMC3840531.

15) Andajani-Sutjahjo S, Ball K, Warren N, Inglis V, Crawford D. Perceived personal, social and environmental barriers to weight maintenance among young women: A community survey. Int J Behav Nutr Phys Act. 2004; 1(15): 1-15. doi: 10.1186/1479-5868-1-15. PMID: 15462679, PMCID: PMC524367.

16) Amiri P, Ghofranipour F, Ahmadi F, Hosseinpanah F, Montazeri A, Jalali-Farahani S, et al. Barriers to a healthy lifestyle among obese adolescents: a qualitive stuady from Iran. Int J Public Health. 2011; 56(2): 181-9. doi: 10.1007/s00038-010-0119-6. PMID: 20143125.

17) Pomerleau J, Lock K, Knai C, McKee M. Interventions designed to increase adult fruit and vegetable intake can be effective: a systematic review of the literature. J Nutr. 2005; 135: 2486-95. PMID: 16177217.

18) Evans GL, McNeil LH, Laufman L, Bowman SL. Determinants of Low-fat Eating Behaviors among Midlife African American Women. J Nutr Educ Behav. 2009; 41(5): 327-33. doi: 10.1016/j.jneb.2008.07.006. PMID: 19717115.

19) French SA, Neumark-Sztainer D, Story M, Jeffery RW. Reducing barriers to participation in weight-loss programs in low-income women. J Am Diet Assoc. 1998; 98(2): 198-200. doi: 10.1016/S00028223(98)00050-9. PMID: 12515425.

20) Alidosti M, Sharifirad GR, Golshiri P, Azadbakht L, Hasanzadeh A, Hemati Z. An investigation on the effect of gastric cancer education based on Health Belief Model on knowledge, attitude and nutritional practice of housewives. Iran J Nurs Midwifery Res. 2012; 17(4): 256-62. PMID: 23833623, PMCID: PMC3702143.

21) Geaney F, Kelly C, Di Marrazzo JS, Harrington JM, Fitzgerald AP, Greiner BA, et al. The effect of complex workplace dietary interventions on employees' dietary intakes, nutrition knowledge and health status: a cluster controlled trial. Prev Med. 2016; 89: 76-83. doi: 10.1016/j.ypmed.2016.05.005. PMID: 27208667.

22) Tamers SL, Beresford SA, Cheadle AD, Zheng Y, Bishop SK, Thompson B. The association between worksite social support, diet, physical activity and body mass index. Prev Med. 2011; 53(1-2): 53-6. doi: 10.1016/j.ypmed.2011.04.012. PMID: 21570422, PMCID: PMC3143200.

23) Beresford SA, Thompson B, Feng Z, Christianson A, McLerran D, Patrick DL. Seattle 5 a Day worksite program to increase fruit and vegetable consumption. Prev Med. 2001; 32: 230-8. doi: 10.1006/pmed.2000.0806. PMID: 11277680.

24) Alinia S, Lassen AD, Krogholm KS, Christensen T, Hels OH, Tetens I. A workplace feasibility study of the effect of a minimal fruit intervention on fruit intake. Public Health Nutr. 2010; 14(8): 1382-7. doi: 10.1017/S1368980010002569. PMID: 21138609.

25) Levin SM, Ferdowsian HR, Hoover VJ, Green AA, Barnard ND. A worksite programme significantly alters nutrient intakes. Public Health Nutr. 2010; 13(10): 1629-35. doi: 10.1017/S136898000999303X. PMID: 20074388 . 